\title{
Coherence automatic adjustment of the optical pulses inside mode-lock fiber laser cavity
}

A. Y. Kokhanovskiy, A. V. Ivanenko, S. K. Kobtsev, S. K. Turitsyn

A. Y. Kokhanovskiy, A. V. Ivanenko, S. K. Kobtsev, S. K. Turitsyn, "Coherence automatic adjustment of the optical pulses inside mode-lock fiber laser cavity," Proc. SPIE 10825, Quantum and Nonlinear Optics V, 1082518 (9 November 2018); doi: $10.1117 / 12.2500786$

SPIE. Event: SPIE/COS Photonics Asia, 2018, Beijing, China 


\title{
Coherence automatic adjustment of the optical pulses inside mode-lock fiber laser cavity
}

\author{
A.Y. Kokhanovskiy ${ }^{a}$, A.V. Ivanenko ${ }^{a}$, , S.K. Kobtsev ${ }^{a}$, S.K. Turitsyn ${ }^{\mathrm{a}, \mathrm{b}}$ \\ ${ }^{a}$ Novosibirsk State University, Pirogova 2, Novosibirsk, 630090 \\ ${ }^{\mathrm{b}}$ Aston Institute of Photonic Technologies, Aston University, B4 7ET, Birmingham, UK
}

\begin{abstract}
In the current work we demonstrate mode-locked fiber laser with automatic adjustment a coherence degree of the output pulses. As a source of the pulses we used 8-figure fiber laser with two amplifying fibers inside both loops of the laser cavity. Such configuration provides various pulsed regimes that have different degree of coherence from fully mode-locked single scale pulses to partly mode-locked double scale pulses. To search a pulsed regime with defined parameters we applied automatic genetic algorithm. To prove the feasibility of the genetic algorithm we applied it to find double - scale pulsed regimes with a fixed envelope duration of $50 \mathrm{ps}$ and the contrast of the coherence peak in range of $0.02-0.5$.
\end{abstract}

Keywords: Double-scale pulses, noise-like pulses, Fiber mode-locked lasers, Machine Learning

\section{INTRODUCTION}

Double - scale (or noise - like) pulses are typical for mode-lock fiber lasers and correspond to partly mode - locked regime of a laser cavity. Such pulses usually are picosecond-scale optical bursts with femtosecond filling structure. Due to amplitude fluctuations double-scale pulses combine a high peak powers with low coherence degree that makes them ideal candidate for optical coherence tomography and spectroscopy applications. Double-scale pulses also have advantage against single scale pulses in frequency conversion processes during supercontinuum generation ${ }^{1}$.

However, at the best of our knowledge, there is no any investigation of an influence of pulse coherence degree on frequency conversion efficiency or during any other applications. It is significant experimental challenge to build robust fiber laser system that provides stable double -scale pulses with variable coherence degree. Moreover, to emphasize coherence degree effect it is important to maintain constant time duration of pulse envelope. Coherence degree of the double-scale pulses can be indirectly measured by amplitude of the narrow central peak in the autocorrelation trace of pulse - so called coherence spike that corresponds to power fluctuation inside optical wave packet ${ }^{2}$.

In this work, we demonstrate special 8-figure mode-locked laser for generation double-scale pulses with variable degree of coherene. Previously, we showed that adding additional amplifying spans of fibre inside laser cavity leads to generation of pulsed regimes with different time duration, energy, optical spectrum and coherence degree ${ }^{3}$. Enormous number of possible pulse regimes allows tuning pulse parameters almost continuously and opens a way for proposed researches. The main drawback of laser system with distributed amplification is a complex dependence of pulsed regimes against gain levels of amplification fibres that demands complex control. Promising way to control output radiation of mode-locked lasers is application of machine learning algorithms. The major part of works related to this topic are devoted to mode-locked fiber lasers based on nonlinear evolution effect since such lasers has more than 6 variable parameters ${ }^{4,5}$. In this work we demonstrate an application of machine learning algorithm at 8-figure fibre laser with distributed amplification.

The goal of this work was to build a laser system with controllable double- scale pulses duration and coherence degree. To overcome a problem with an adjustment of the pulse parameters we applied genetic algorithm that is based on the principle of artificial selection. To prove the feasibility of the genetic algorithm we applied it to find double - scale pulses with a fixed envelope duration at $50 \mathrm{ps}$ and the contrast of the coherence peak in range of $0.02-0.5$.

\section{EXPERIMENTAL SETUP}

The scheme of 8-figure mode-locked fiber laser cavity is presented at (Fig.1). Both loops of the laser resonator contain amplifying sections of $2.5 \mathrm{~m}$ long, which are pumped by independent multimode laser diodes with an optical

Quantum and Nonlinear Optics V, edited by Qihuang Gong, Guang-Can Guo,

Byoung S. Ham, Proc. of SPIE Vol. 10825, 1082518 · (c) 2018 SPIE

CCC code: $0277-786 \mathrm{X} / 18 / \$ 18 \cdot$ doi: $10.1117 / 12.2500786$ 
power of up to $4 \mathrm{~W}$ at a wavelength of $978 \mathrm{~nm}$. The right loop contains a high-power Faraday rotator, which provides unidirectional propagation of optical radiation. The loops are connected by a coupler $40 / 60$. The output radiation is released from $70 \%$ port of the fiber coupler.



Figure 1: Schematic of 8 -figure fiber laser with two active stretches of fiber in both loops.

Measuring a pulse autocorrelation function gives information of its duration and coherence degree. It is well-known, that double-scale pulses have a coherence spike at a center of autocorrelation function (ACF) and its height indicates a degree of coherence of mode-locking regime. To measure a contrast of coherence spike regards to ACF envelope we applied low-pass Butterworth filter that cuts coherence spike. Then we take a full-width half maximum of filtered ACF as duration of ACF envelope and calculate a ratio between maximums of unfiltered and filtered ACF as a contrast of coherence spike (Fig. 2.).

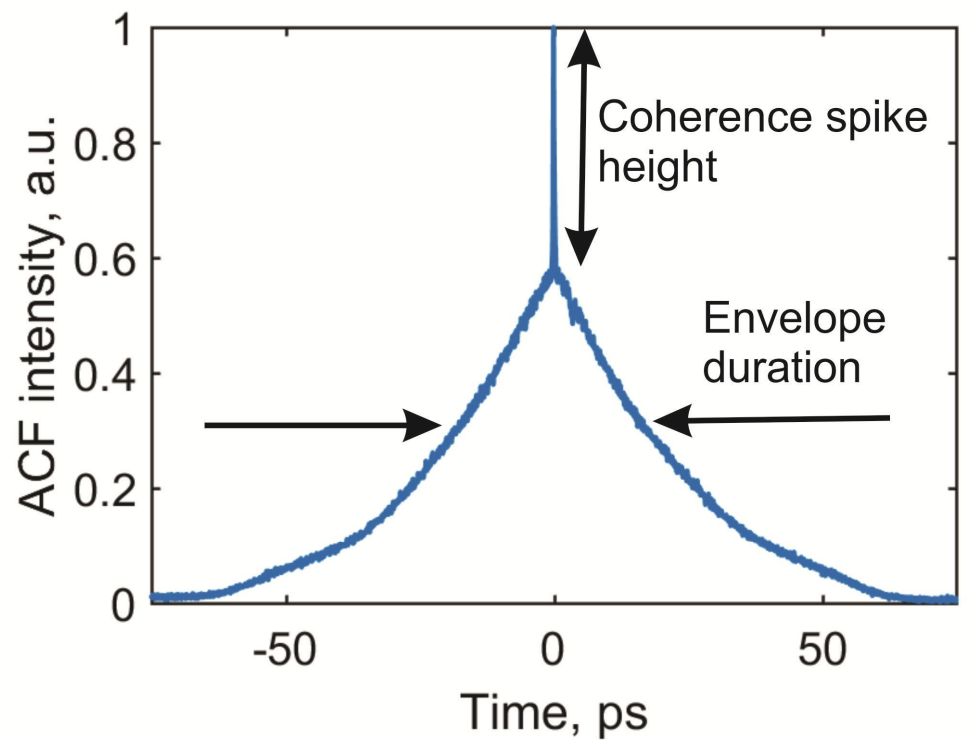

Figure 2: Typical ACF trace of double scale pulse

Using A.P.E. PulseCheck autocorrelator we built a map of pulsed regimes that shows their complex distribution depending on currents applied to pump diodes (Fig.3). 


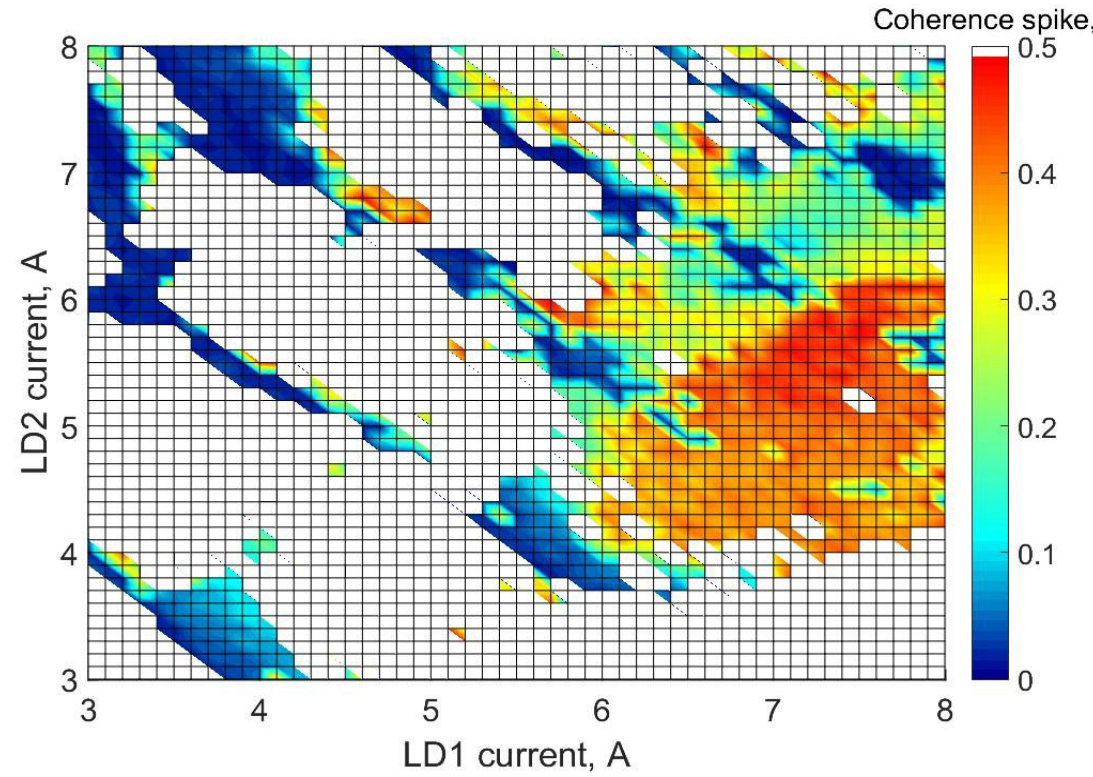

Figure 3: Map of coherence peak contrast of the pulsed regimes.

We filtered regimes that do not correspond to mode-locked regime and denoted them by white color. We chose a contrast of radio-frequency spectrum in the vicinity of fundamental mode of laser cavity as a criterion of mode-locking regime (Fig.4.). Regimes with radio-frequency contrast more than $40 \mathrm{~dB}$ were considered as mode-locked regimes.



Figure 4: Radio-frequency spectrum of the double-scale pulse b) Typical optical spectrum of the double-scale pulse

\section{GENETIC ALGORITHM}

Proposed laser configuration provides almost continuous variation of any parameter of pulsed regime; therefore genetic algorithm is a convenient method for picking up pulsed regime with specified parameters. The algorithm of genetic search is based on the principle of artificial selection (Fig.5.).

Individual of the population is a pulsed regime having genes - values of pumping diodes currents. Each individual has unique set of parameters: contrast of radio-frequency spectrum of fundamental mode, average power, duration of autocorrelation function, contrast of coherence spike. Using these values one can construct a fitness function that genetic 
algorithm has to optimize. Fitness function should be selected such way that its minimum value corresponds to pulsed regime with the closest desirable parameters.

Genetic algorithm starts with an initialization of population assigning random set of currents of pumping diodes. Then pulse parameters are measured for each individual in population. We normalize these parameters by the maximum values in the population in order to have equal weights of each parameter in the fitness function, thus each value lies in the range from 0 to 1 . After sorting the population by the value of the fitness function, individuals are mated by random genes mixing. Some of the individuals with the highest value of the fitness function - the elite part is not changed. To avoid algorithm to clamp on the local maximum of the fitness function, part of the population is exposed by mutation - a random change of genes. The algorithm cycle can be terminated when the change in the objective function for several cycles does not exceed the threshold value.

To configure the algorithm, the number of individuals in the population, fraction of elite individuals, fraction of mutated individuals is used. These parameters are selected empirically and in our case number individuals in population $\mathrm{N}_{\text {pop }}=30$, Elite fraction $\mathrm{F}_{\text {elit }}=0.2$, Mutation fraction $\mathrm{F}_{\mathrm{mut}}=0.2$.

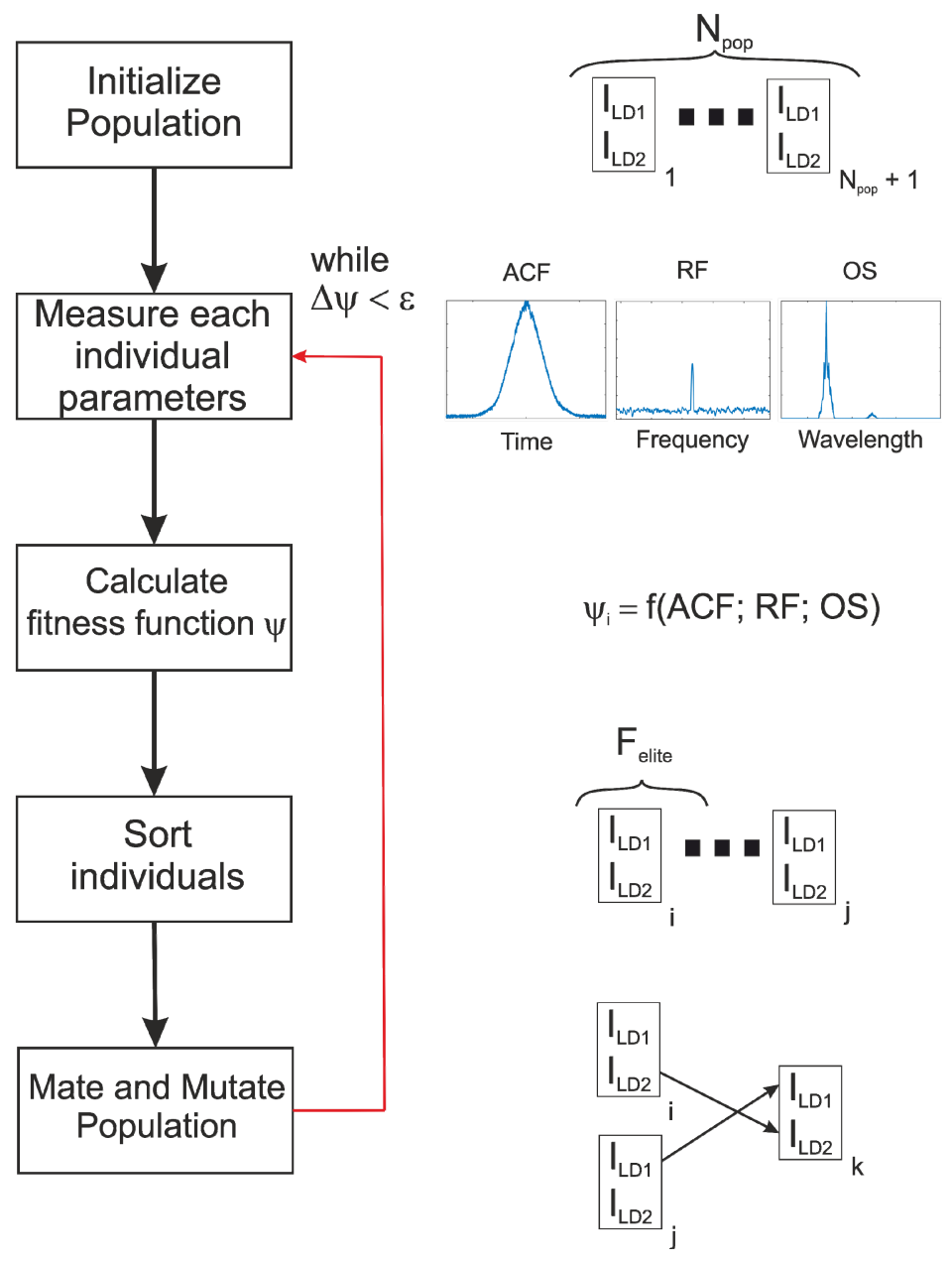

Figure 5: Scheme of genetic algorithm applied to laser system

\section{RESULTS}

To find double-scale pulses with predetermined parameters the fitness function was calculated as the square of the difference between the parameters of the current pulsed regime and the desired one. To search for double-scale pulses with envelope duration $\mathrm{ACF}_{\text {ref }}$ and coherence spike contrast $\mathrm{Coh}_{\text {ref }}$, the following function was used: 


$$
\psi_{4}=\left(A C F_{\text {ow }}-A C F_{r q f}\right)^{2}+\left(C o h_{o w p}-C_{O H} h_{r e f}\right)^{2}
$$

We fixed $\mathrm{ACF}_{\text {ref }}$ equal $50 \mathrm{ps}$ and put different values of $\mathrm{Coh}_{\text {ref }}$ in range of 0.02-0.5. Genetic algorithm was applied for each value of $\mathrm{Coh}_{\text {ref }}$ separately by changing the values in fitness function. The resulting pulses are demonstrated in Fig.6.

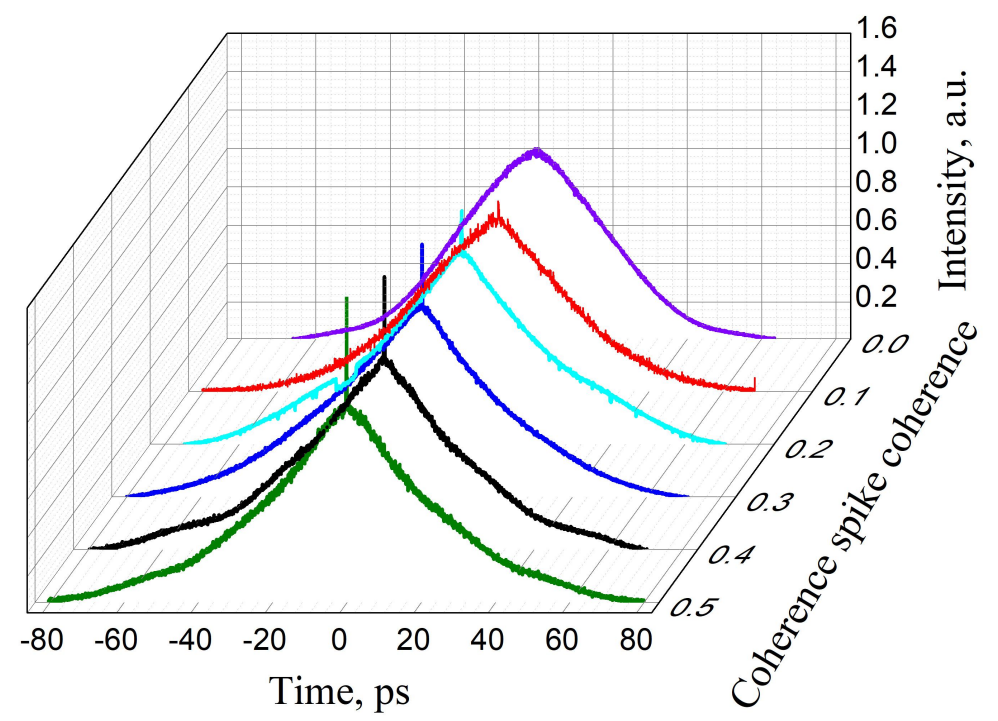

Figure 6: Double-scale pulses of a laser cavity with a constant envelope duration of 50 ps and a different contrast of the coherence peak in the range from 0 to 0.5

In general, it took $10-15$ iteration of genetic algorithm to find pulses with predetermined parameters. Proceeding fifteen iterations demanded acquisition of 450 experimental measurements that lasted 2 second each, so the average total time of searching pulsed regime does not exceed 15 minutes.

\section{CONCLUSIONS}

We demonstrated the self-tuning 8-figure mode-locked fibre laser generating double-scale pulse with variable coherence degree. Application of genetic algorithm provides reliable adjustment of pulse parameters on demand. We believe that such approach could enrich investigations of double-scale pulses features in different scientific and technological applications.

\section{ACKNOWLEDGMENTS}

Work of A.Y. Kokhanovskiy was funded by RFBR according to the research project № 18-32-00560 . Work of S.K. Turitsyn and A.V. Ivanenko was supported by the Russian Science Foundation (Grant No. 17-72-30006). Work of S.M. Kobtsev was funded by RFBR according to the research project № 16-02-00104. 


\section{REFERENCES}

[1] Kobtsev, S., Kukarin, S., Smirnov, S. and Ankudinov, I., "Cascaded SRS of single- and double-scale fiber laser pulses in long extra-cavity fiber," Opt. Express 22(17), 20770 (2014).

[2] Kobtsev, S., Smirnov, S. and Kukarin, S., [Double-scale Pulses Generated by Mode-locked Fibre Lasers and Their Applications] (2016).

[3] Kobtsev, S., Ivanenko, A., Kokhanovskiy, A. and Smirnov, S., "Electronic control of different generation regimes in mode-locked all-fibre F8 laser," Laser Phys. Lett. 15(4) (2018).

[4] Woodward, R. I. and Kelleher, E. J. R., "Towards 'smart lasers': self-optimisation of an ultrafast pulse source using a genetic algorithm,” Nat. Publ. Gr.(November), 1-9 (2016).

[5] Baumeister, T., Brunton, S. L. and Kutz, J. N., "Deep Learning and Model Predictive Control for Self-Tuning Mode-Locked Lasers," 1-10 (2017). 